\title{
Coexistent quantum and classical aspects of magnetization plateaux in alternating-spin chains
}

\author{
Tôru Sakai \\ Faculty of Science, Himeji Institute of Technology, Ako, Hyogo 678-1297, Japan \\ Shoji Yamamoto \\ Department of Physics, Okayama University, Tsushima, Okayama 700-8530, Japan
}

(October 28, 2018)

\begin{abstract}
Magnetization process of ferrimagnetic Heisenberg chains of alternating spins are theoretically studied. The size scaling analysis with the exact diagonalization of finite systems for $(S, s)=(3 / 2,1)$ and $(2,1)$ indicates a multi-plateau structure in the ground-state magnetization curve for $S$ and $s$ $>1 / 2$. The first plateau at the spontaneous magnetization can be explained by a classical origin, that is the Ising gap. In contrast, the second or higher one must be originated to the quantization of the magnetization. It is also found that all the $2 s$ plateaux, including the classical and quantum ones, appear even in the isotropic case with no bond alternation.
\end{abstract}

PACS numbers: $75.10 . \mathrm{Jm}, 75.40 \mathrm{Mg}, 75.30 . \mathrm{Kz}$

\section{INTRODUCTION}

Alternating spin chains with antiferromagnetic interactions have been attracting a lot of current interest. They behave like a gapped antiferromagnet based on the optical mode of the low-lying excitation, while they also exhibit a ferromagnetic aspect characterized by a spontaneous magnetization. The coexistence of the two aspects gives rise to various interesting crossover phenomena at low temperatures.(Yamamoto 2000) However, few quantum aspects have ever been reported for the systems. Then we consider an interesting phenomenon caused by a quantum mechanism, called quantization of magnetization, on the stage of the quantum ferrimagnetic chains. It would be observed as a plateau in the ground-state magnetization curve. Recently many theoretical (Oshikawa et al. 1997, Totsuka 1998, Tonegawa et al. 1996, Cabra et al. 1997, Cabra and Grynberg 1999 and Sakai and Takahashi 1998) and experimental (Narumi et al. 1998 and Shiramura et al. 1998) studies suggested the realization of the magnetization plateaux in various systems.

The previous works (Yamamoto and Sakai 1998 and Sakai and Yamamoto 1998) by the present authors indicated an important role of the quantum fluctuation to stabilize the plateau against the planar anisotropy in the ferrimagnetic chain. It results in the existence of the plateau even in the $X Y$ model of the mixed spins 1, and $1 / 2$. On the other hand, the classical mixed spin systems have the same plateau in the isotropic case. It implies that the plateau is originally based on a classical mechanism, although it is stabilized by a quantum effect. Thus it is difficult to say that the plateau symbolizes the quantum nature of the ferrimagnetic chains. In the present paper, other plateaux, essentially based on a quantum mechanism, are revealed to coexist with the above mentioned classical plateau, when both spins are lager than $1 / 2$.

\section{QUANTUM AND CLASSICAL PLATEAUX}

The recent exact treatment (Oshikawa et al. 1997) for general quantum spin chains suggested that a magnetization plateau can appear based on the quantization of the magnetization under the condition,

$$
S_{\text {unit }}-m=\text { integer, }
$$

where $S_{\text {unit }}$ and $m$ are the total spin and magnetization per unit cell. Note that the relation (11) is only a necessary condition. Thus it doesn't guarantee the existence of the plateau nor specify any mechanisms of its formation. The condition (1) is still valid for the mixed spin chains of $S$ and $s(S>s)$, described by the Hamiltonian

$\mathcal{H}=\sum_{j=1}^{N}\left[(1+\delta)\left(\boldsymbol{S}_{j} \cdot \boldsymbol{s}_{j}\right)_{\alpha}+(1-\delta)\left(\boldsymbol{s}_{j} \cdot \boldsymbol{S}_{j+1}\right)_{\alpha}-H\left(S_{j}^{z}+s_{j}^{z}\right)\right]$

with $(\boldsymbol{S} \cdot \boldsymbol{s})_{\alpha}=S^{x} s^{x}+S^{y} s^{y}+\alpha S^{z} s^{z}$. In the isotropic case $(\alpha=1)$ with no bond alternation $(\delta=0)$, the system has a spontaneous magnetization $m_{s} \equiv S-s$. Because of the antiferromagnetic gap the ground state with $m_{s}$ is so stable against the excitation increasing $m$ that there appears a magnetization plateau at $m=m_{s}$. (Kuramoto 1998) The previous works (Yamamoto and Sakai 1998 and Sakai and Yamamoto 1998) on the most quantized system $(S, s)=(1,1 / 2)$ suggested that the quantum fluctuation stabilizes the plateau against the $X Y$ like anisotropy $(\alpha<1)$ and the plateau phase extends to the Kosterlitz-Thouless phase boundary in the ferromagnetic region $(\alpha<0)$. However, this plateau phase also includes the Ising limit $(\alpha \rightarrow \infty)$ without any other boundaries. Thus it is difficult to identify some quantum effects on the plateau formation, because it cannot be clearly distinguished from the Ising gap based on 
a classical mechanism. In fact the classical spin (vector) model with the same amplitudes $(S, s)=(1,1 / 2)$ described by the same Heisenberg Hamiltonian (2) also has a plateau at $m=m_{s}$ for $\alpha=0$. Then the plateau at $m_{s}$ should be called a classical plateau. On the other hand, the condition of the quantization (11) suggests that some other plateaux can appear at higher magnetization $m=S-s+1, S-s+2, \cdots, S+s-1$ for $S>s>1 / 2$. These higher plateaux can never be explained by any classical mechanisms, because they cannot appear in the Ising model or classical Heisenberg model. Thus they should be called quantum plateaux, if they really appear. In the following sections, we perform some theoretical analyses for the systems of $(S, s)=(3 / 2,1)$ and $(2,1)$ to justify the coexistence of the quantum and classical plateaux in the case of $S>s>1 / 2$.

\section{LOW-LYING EXCITATIONS}

The optical mode of the low-lying excitations characterizes the feature of the initial plateau at $m_{s}$. In Fig. 1 we show the excitation spectra of the systems $(\mathrm{a})(3 / 2,1)$ and $(\mathrm{b})(2,1)$ for $\alpha=1$ and $\delta=0$, derived from the three methods; the quantum Monte Carlo simulation (QMC), the modified spin wave theory and the perturbation from the decoupled dimer. The first one gives the most precise results and the last one is based on the dimer state described as $\prod_{j}\left(A_{j}^{\dagger}\right)^{S-s}\left(A_{j}^{\dagger} b_{j}^{\dagger}-B_{j}^{\dagger} a_{j}^{\dagger}\right)^{2 s}|0\rangle$, making use of the Schwinger boson representation:

$$
\begin{aligned}
& S_{j}^{+}=A_{j}^{\dagger} B_{j}, \quad S_{j}^{z}=\frac{1}{2}\left(A_{j}^{\dagger} A_{j}-B_{j}^{\dagger} B_{j}\right), \\
& s_{j}^{+}=a_{j}^{\dagger} b_{j}, \quad s_{j}^{z}=\frac{1}{2}\left(a_{j}^{\dagger} a_{j}-b_{j}^{\dagger} b_{j}\right),
\end{aligned}
$$

The excitation spectrum of each system has two branches characterizing the ferromagnetic (lower) and antiferromagnetic (upper) features, as well as the system $(1,1 / 2)$. The calculated curves suggest that the spin wave is more suitable than the decoupled dimer to describe the behavior of the optical branch around its bottom $(k=0)$. It implies that the classical picture (spin wave excitation from the Néel order) is more effective than the quantum one (dimer-breaking excitation) to explain the origin of the initial plateau, as expected in the above argument.
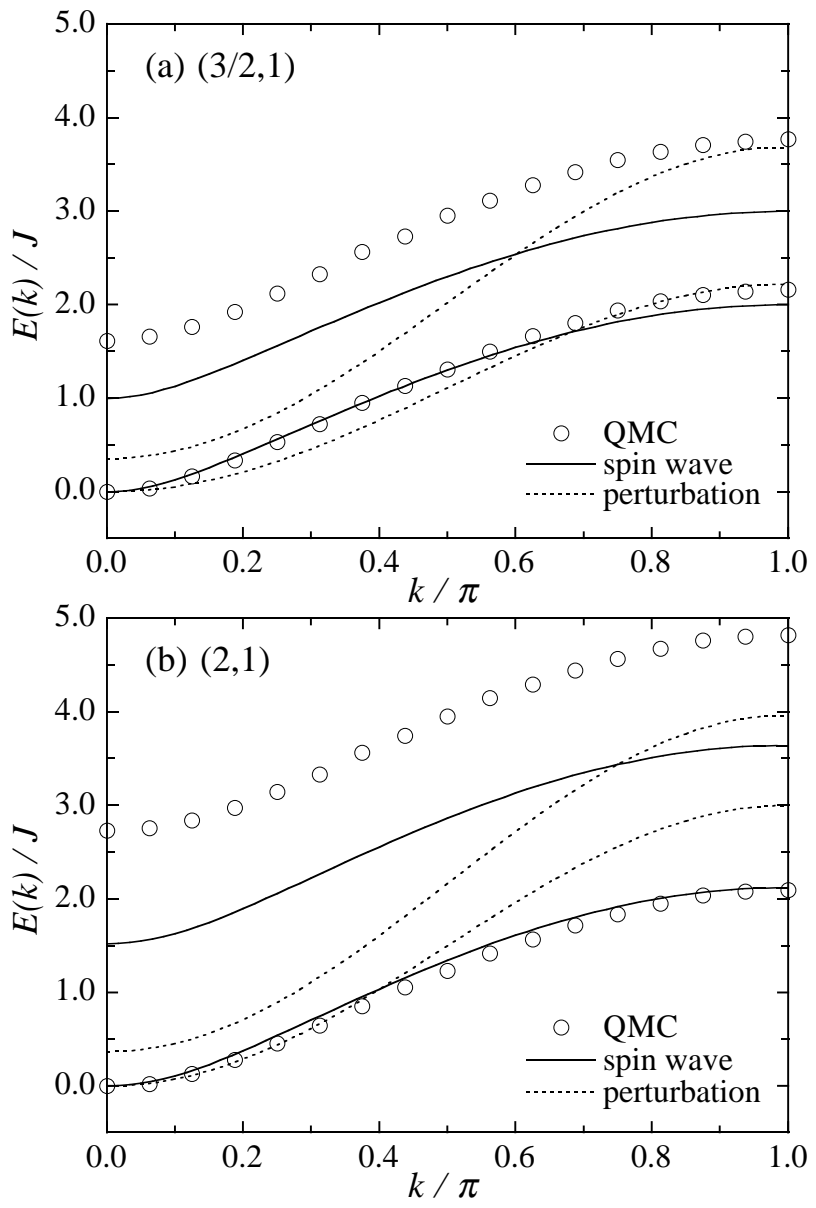

FIG. 1. Low-lying excitation spectra calculated by the quantum Monte Carlo simulation (QMC), the spin wave theory and the perturbation from the decoupled dimer for (a) $(3 / 2,1)$ and (b) $(2,1)$.

\section{VARIATIONAL APPROACH}

According to the condition of the quantization (11), the mixed-spin chains of $(3 / 2,1)$ and $(2,1)$ possibly have two plateaux at $m=m_{s}$ and $m_{s}+1$. In order to characterize these plateaux, we introduce a variational wave function for the ground state of the model (2) as

$$
\begin{gathered}
|\mathrm{g}\rangle= \\
c_{\mathrm{N}} \prod_{j=1}^{N}\left(A_{j}^{\dagger}\right)^{2 S}\left(b_{j}^{\dagger}\right)^{2 s}|0\rangle \\
+\sum_{l=0}^{2 s} c_{\mathrm{VB}}^{(l)} \prod_{j=1}^{N}\left(A_{j}^{\dagger}\right)^{2 S-l}\left(a_{j}^{\dagger}\right)^{2 s-l}\left(A_{j}^{\dagger} b_{j}^{\dagger}-B_{j}^{\dagger} a_{j}^{\dagger}\right)^{l}|0\rangle,
\end{gathered}
$$

where $c_{\mathrm{N}}$ and $c_{\mathrm{VB}}^{(l)}$ are the mixing coefficients. Using the variational wave function, the ground-state phase diagram in the $H \delta$ plane is obtained, as shown in Figs. 2 (a) and (b) for $(3 / 2,1)$ and $(2,1)$, respectively, where we restrict us on the Heisenberg point $(\alpha=1)$. On the first step of the magnetization process for each system, there exists a crossover point $\delta_{c}$ between the Néel $(\mathrm{N})$ and double-bond dimer (DBD) states. In contrast, the second step toward the saturation $(\mathrm{S})$ is always the single-bond dimer (SBD) state. These two steps before the saturation 
are expected to characterize the two plateaux. Thus the first plateau should be based on the classical Néel order, while the second one on the quantum Valence-Bond-Solid state, as far as we consider the case of small $\delta$.
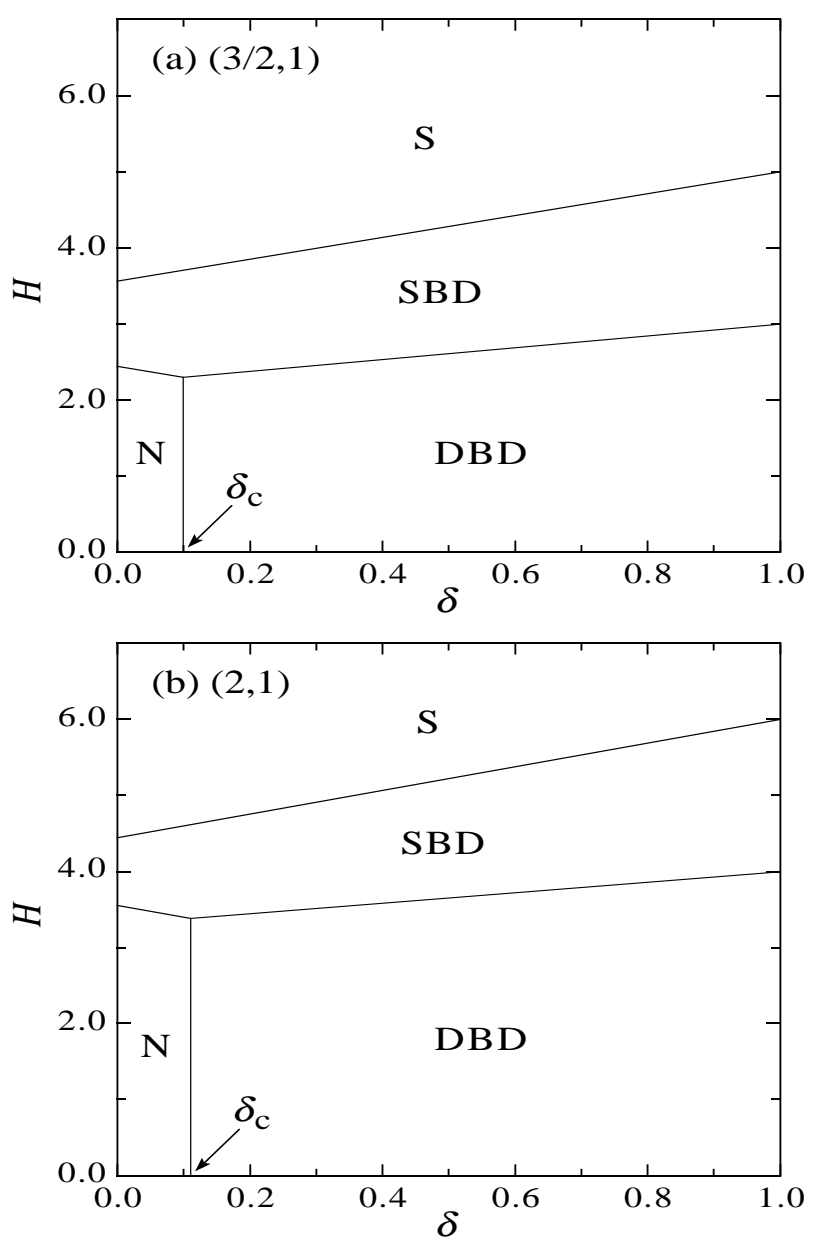

FIG. 2. The variational ground-state phase diagrams on the $\delta H$-plane with $\alpha=1$ for $(\mathrm{a})(3 / 2,1)$ and $(\mathrm{b})(2,1)$. Phases are denoted as N: Néel, DBD: double-bond dimer, SBD: single-bond dimer and S: saturation, respectively.

\section{PHASE DIAGRAMS}

In order to confirm the coexistence of the two plateaux even for $\alpha=1$ and $\delta=0$, we perform a size scaling analysis with the exact diagonalization of finite systems up to $N=12$ to present the phase diagrams in the $\delta \alpha$ plane. $E(N, M)$ denotes the lowest energy in the subspace with a fixed magnetization $M$ for the Hamiltonian (2) without the Zeeman term. The upper and lower bounds of the external field which induces the groundstate magnetization $M$ are expressed as $H_{ \pm}(N, M)=$ $\pm E(N, M \pm 1) \mp E(N, M)$. The length of the plateau with the unit-cell magnetization $m \equiv M / N$ is obtained as $\Delta_{N}(m)=H_{+}(N, M)-H_{-}(N, M)$. The quantity $\Delta_{N}(m)$ also corresponds to the sum of the two excitation gaps for increasing and reducing the magnetization,
respectively.(Sakai and Takahashi 1998) Thus the scaled quantity $N \Delta_{N}(m)$ is a good probe of the plateau. No size dependence means that the system is gapless. The scaled quantity of the system $(3 / 2,1)$ at (a) $m=1 / 2$ and (b) $m=3 / 2$ is shown as a function of $\alpha$ for $\delta=0$ in Fig. 3. Fig. 3 (a) clearly shows that opening plateau around $\alpha=1$ vanishes at some critical value $\alpha_{c}$ for $m=1 / 2$ and a gapless phase lies in the region of $\alpha<\alpha_{c}$. The scaled gap in Fig. 3 (b) also indicates the existence of the second plateau around $\alpha=1$, although the size dependence is much smaller than that of the first plateau. It implies that the second plateau is much smaller than the first one.

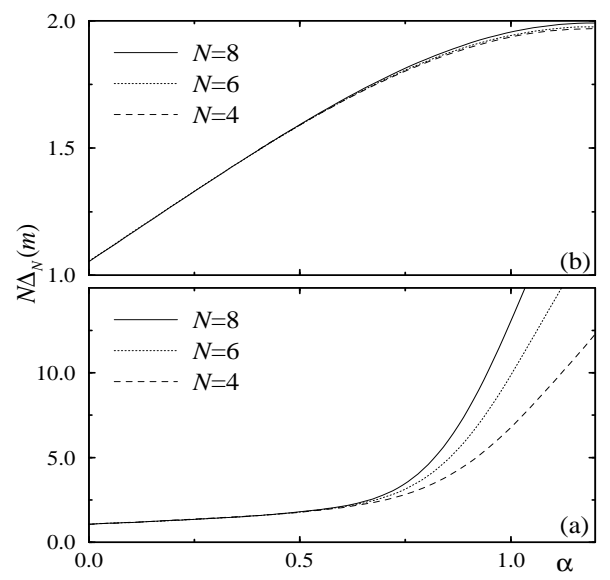

FIG. 3. Scaled quantity $N \Delta_{N}(m)$ versus $\alpha$ at $m=\frac{1}{2}$ (a) and $m=\frac{3}{2}$ (b) in the case of $(S, s)=\left(\frac{3}{2}, 1\right)$.

To investigate the critical property around $\alpha_{c}$, we use the size scaling formula based on the conformal field theory (Cardy 1984, Blöte et al. 1986 and Affleck 1986)

$$
\frac{1}{N} E(N, M) \sim \epsilon(m)-\frac{\pi v_{\mathrm{s}} c}{6 N^{2}},
$$

and

$$
\Delta_{N}(m) \sim \frac{\pi v_{\mathrm{s}} \eta}{N}
$$

with the following notations: $\epsilon(m)$ is the ground state energy of the bulk system; $v_{\mathrm{s}}$ is the sound velocity derived from the derivative of the dispersion curve at $k=0 ; c$ is the central charge; $\eta$ is the critical exponent defined by the spin-correlation function $\left\langle\tilde{s}_{0}^{x} \tilde{s}_{r}^{x}\right\rangle \sim r^{-\eta}$, where $\tilde{\mathbf{s}}_{i}$ is some relevant spin operator. They are valid at gapless points. The calculated $c$ and $\eta$ of the systems $(3 / 2,1)$ and $(2,1)$ at $m=m_{s}$ and $m=m_{s}+1$ indicate the following properties: as $\alpha$ decreases from 1 with fixed $\delta$, the first and second plateaux vanish at the different Kosterlitz-Thouless critical points $\alpha_{c 1}$ and $\alpha_{c 2}$, respectively, where $\eta$ is $1 / 4$ in common; the gapless spin fluid phase characterized by $c=1$ lies in the region $\alpha<\alpha_{c 1}$ $\left(\alpha_{c 2}\right)$ at $m=m_{s}\left(m=m_{s}+1\right)$. The universality of the phase boundary of both plateaux are the same as that of 
the unique plateau of the system $(1,1 / 2)$ (Yamamoto and Sakai 1999 and Sakai and Yamamoto 1999). Thus we determine the gapless-plateau phase boundary by $\eta=1 / 4$ for both plateaux. Thus-obtained phase boundaries for the first and second plateaux are shown together as solid lines in Figs. 4 (a) and (b) for the systems $(3 / 2,1)$ and $(2,1)$, respectively. The phase diagrams obviously turn out the coexistence of the first (classical) and second (quantum) plateaux even at the most symmetric point $(\alpha=1$ and $\delta=0)$. They also exhibit an interesting feature; the quantum plateau phase is larger than the classical one $\left(\alpha_{c 1} \leq \alpha_{c 2}\right.$ independently of $\left.\delta\right)$. It implies that the quantum plateau is more stable than the classical one against the planar anisotropy.
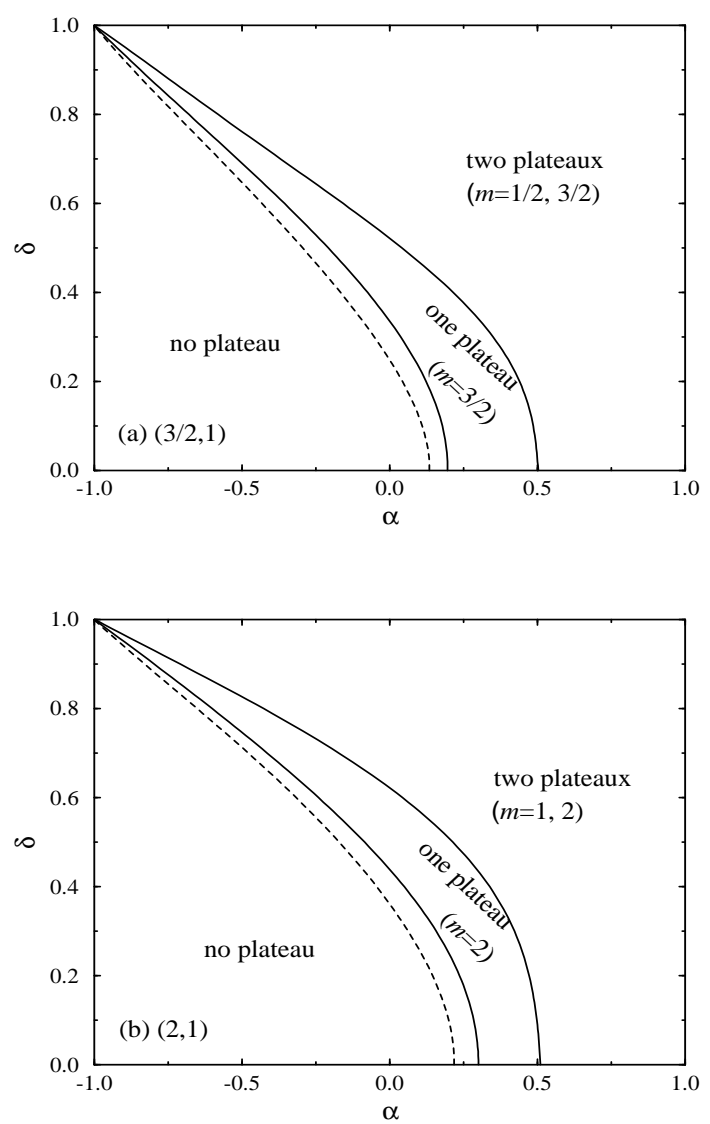

FIG. 4. The ground-state phase diagrams on the $\alpha \delta$-plane for $(\mathrm{a})(3 / 2,1)$ and $(\mathrm{b})(2,1)$. Solid lines are the phase boundaries determined by $\eta=1 / 4$. Dashed lines are the boundaries of the second plateaux determined by the level spectroscopy. A small difference between the two results for the second plateaux is due to the logarithmic size correction which should appear in the former method.

Such a slight size dependence of the scaled quantity $N \Delta_{N}(m)$ for the second plateau as shown in Fig. 3 (b) might make us doubt its existence for $\alpha=1$ and $\delta=0$. Then we perform another analysis, called level spectroscopy (Okamoto and Nomura 1992 and Nomura
1995), to convince of the existence of the second plateau. It is one of the most precise methods to estimate the Kosterlitz-Thouless phase boundary. Since the method detects the boundary as a level crossing point of the two relevant excitation gaps with the same scaling dimension, the result does not suffer from the dominant logarithmic size correction, which is quite serious for the Kosterlitz-Thouless transition. For the second plateau at $m=S-s+1$, the two relevant gaps are given by

$$
\begin{gathered}
\Delta_{0} \equiv E_{2}\left(L, M_{2}\right)-E\left(L, M_{2}\right) \\
\Delta_{4} \equiv\left[E\left(L, M_{2}+4\right)+E\left(L, M_{2}-4\right)-2 E\left(L, M_{2}\right)\right] / 2,
\end{gathered}
$$

where $E_{2}(L, M)$ is the second eigenvalue in the same subspace as $\mathrm{E}(\mathrm{L}, \mathrm{M})$ and $M_{2}$ is defined as $M_{2} \equiv(S-s+1) N$. The two excitations have a common scaling dimension 2. The calculated $\Delta_{0}$ and $\Delta_{4}$ of the system $(3 / 2,1)$ for $\delta=0$ are plotted versus $\alpha$ in Fig. 5 . It suggests that the phase boundary is easily determined as a crossing point, almost independent of the system size. Thus-obtained boundaries for the second plateaux are shown as dashed curves in Figs. 4 (a) and (b). The results have a little deviation from the boundaries by $\eta=1 / 4$, because the latter includes the logarithmic size correction. Anyway they lead to the same conclusion; the coexistence of the classical and quantum plateaux for $\alpha=1$ and $\delta=0$.

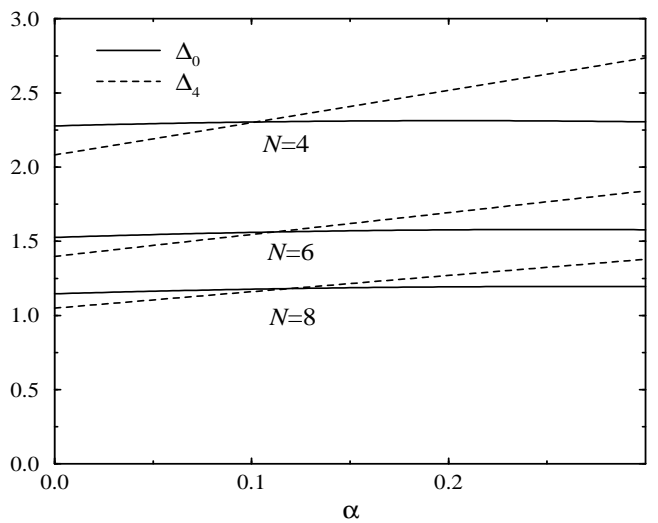

FIG. 5. $\quad \Delta_{0}$ and $\Delta_{4}$ of the system $(3 / 2,1)$ with $\delta=0$ versus $\alpha$. It indicates that the crossing point of the two gaps is almost independent of the system size.

The Néel-dimer crossover point in the first plateau indicated by the variational method in the previous section is not detected as any phase boundaries by these numerical analyses. It suggests that the Néel and dimer pictures cannot be distinguished clearly for the first plateau. In fact it is trivially revealed that in the $\delta \alpha$ phase diagram at $m=m_{s}$ the isotropic dimer point $(\alpha=1$ and $\delta=1)$ is connected to the Ising limit $(\alpha \rightarrow \infty$ and $\delta=0)$ via the 
Ising dimer limit $(\alpha \rightarrow \infty$ and $\delta=1)$ through no phase transition or crossover. It implies that the first plateau always bears an aspect of the Ising gap even for large $\delta$.
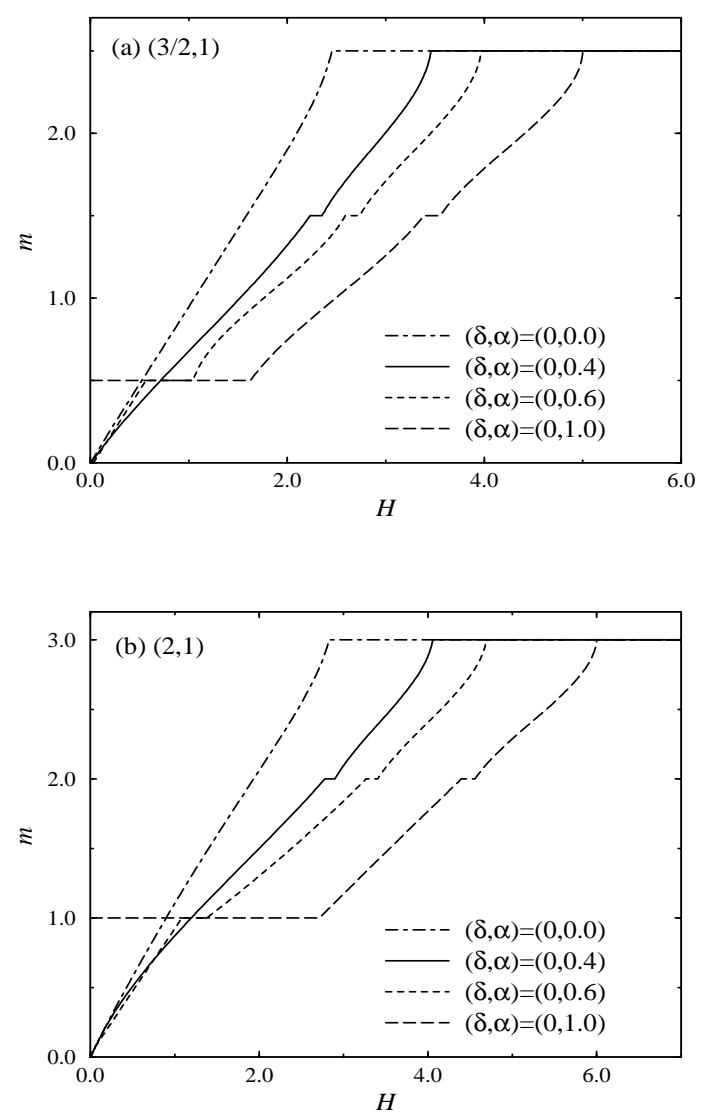

FIG. 6. The ground-state magnetization curves of the quantum system with $\delta=0$ at various values of $\alpha$ for (a) $(3 / 2,1)$ and (b) $(2,1)$.

\section{MAGNETIZATION CURVE}

Finally we present the ground state magnetization curve in several cases of the systems $(3 / 2,1)$ and $(2,1)$. The curve is given by extrapolating $H_{ \pm}(N, M)$ to the thermodynamic limit using the size scaling (Sakai and Takahashi 1998) based on the conformal field theory at gapless points and the Shanks transformation for plateaux. We show only the results of a suitable polynomial fitting to thus-obtained points, in Figs. $6(\delta=0)$ and $7(\delta=0.4)$, where the labels (a) and (b) indicate the systems $(3 / 2,1)$ and $(2,1)$, respectively. They visualize the coexistence of the classical and quantum plateaux at the Heisenberg point. These results also justify the above mentioned feature; the quantum plateau is smaller at the Heisenberg point but more stable against the $X Y$ like anisotropy, rather than the cassical one. Therefore, if these systems lose a plateau due to the anisotropy, only the quantum one would survive. In order to clarify the difference in the mechanism of the gap formation between the first and second plateaux, we also present the magnetization curves of the classical Heisenberg spin systems described by the same Hamiltonian (2) with the same amplitudes $(S, s)=(3 / 2,1)$ and $(2,1)$ in Figs. 8 (a) and (b), respectively. (The results are independent of $\delta$.) The classical systems clearly have the first plateau in the isotropic case, while there appears no plateau corresponding to the second one. It also supports the quantum nature of the second plateau. These plateaux of the classical systems vanishes even for a slight anisotropy; $\alpha_{c}=0.980$ and 0.943 for $(S, s)=(3 / 2,1)$ and $(2,1)$, repectively. In comparison with these critical values, the phase boundaries in the quantum systems in Figs. 4 (a) and (b) imply that quantum fluctuation stabilies even the first plateau. Thus, in geneal, the quantum effect is expected to toughen every field-induced gap against the planar anisotropy. Nevertheless the first and second plateaux should be distinguished, because the former appears even in the classical limit, while the latter doesn't exist until the spin is quantized.
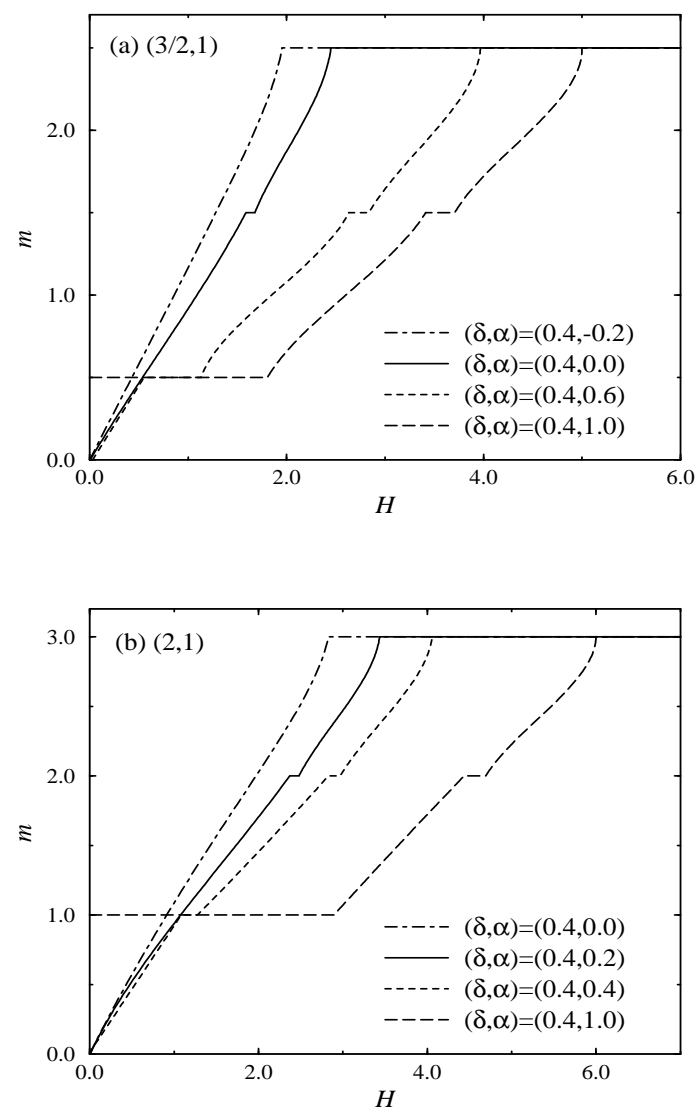

FIG. 7. The ground-state magnetization curves of the quantum system with $\delta=0.4$ at various values of $\alpha$ for (a) $(3 / 2,1)$ and $(b)(2,1)$. 

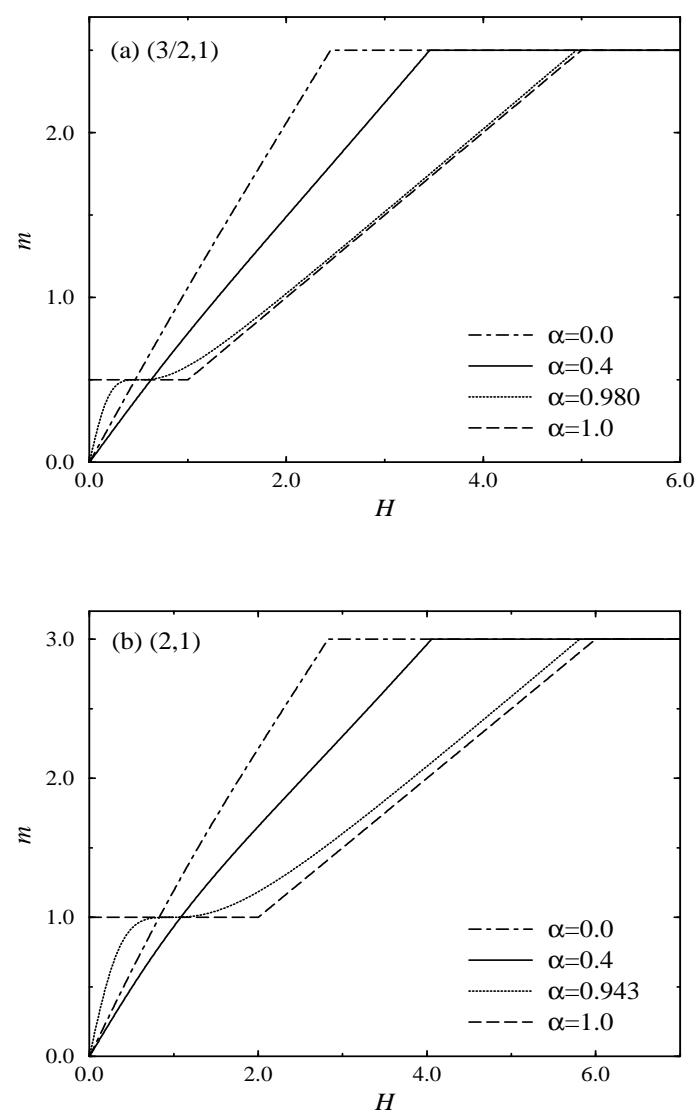

FIG. 8. The ground-state magnetization curves of the classical system at various values of $\alpha$ for $(a)(3 / 2,1)$ and (b) $(2,1)$.

\section{CONCLUDING REMARKS}

The above investigations turn out the coexistence of the classical and quantum plateaux in the ground-state magnetization curve of the mixed spin chains of $(3 / 2,1)$ and $(2,1)$. The conclusion is easily generalized for $(\mathrm{S}, \mathrm{s})$ $(S>s>1 / 2)$; the magnetization curve has $2 s$ plateaux and only the initial one is based on a classical mechanism, while the other ones originate in quantum correlations.

In most previous works on the magnetizaion plateau the gap formation were based on the bond polymerization. (Totsuka 1998, Tonegawa et al. 1996, Cabra et al. 1997, Cabra and Grynberg 1999) In contast, the present proposal of the plateau in ferrimagnetic chains is a pioneering trial to explore a novel mechanism of the fieldinduced gap associated to the spin polymerization. The bimetallic chains such as $\mathrm{MM}^{\prime}(\mathrm{pbaOH})\left(\mathrm{H}_{2} \mathrm{O}\right)_{3} \cdot \mathrm{nH}_{2} \mathrm{O}$ (Karn 1989 and Kahn et al. 1995) are good candidates to realize the spin plymerization. Unfortunately, most of them are in the case of $\mathrm{M}^{\prime}=\mathrm{Cu}$, that is $s=1 / 2$. In fact a few compounds with other metals were also synthesized, for example, $\mathrm{MM}^{\prime}(\mathrm{EDTA}) 6 \mathrm{H}_{2} \mathrm{O} \quad\left(\mathrm{MM}^{\prime}=\mathrm{CoNi}, \quad \mathrm{MnCo}\right.$ and $\mathrm{MnNi}$. However, the case of $\mathrm{MM}^{\prime}=\mathrm{CoNi}$ yields $(S, s)=(1(\mathrm{Ni}), 1 / 2(\mathrm{Co}))$ (Drillon et al. 1985) and $\mathrm{MnCo}$ a large Ising-like anisotropy. (Drillon et al. 1986) Thus they are not any suitable stages to search for the quantum plateau. Among the series of bimetallic chains, the most suitable compound might be $\mathrm{MnNi}(\mathrm{EDTA}) 6 \mathrm{H}_{2} \mathrm{O}$, which is well described by the $1 \mathrm{D}$ spin-alternating Heisenberg model of $(5 / 2,1)$. (Drillon et al. 1986) The magnetization measurement on it would be interesting to investigate a possible quantum plateau at $m=5 / 2$, as well as the classical one at $m=3 / 2$.

One of the most important remarks in the present work is the coexistence of the quantum and classical plateaux even at the most symmetric point $(\alpha=1$ and $\delta=0)$. In order to examine it, compounds consisting of metals and stable organic radicals (Caneschi et al. 1989 and Markosyan et al. 1998) might be a more ideal stage, because the organic radicals lead to entirely isotropic spin systems, rather than the bimetallic chains with inevitable Ising-like anisotropy. The metal-radical complex also has a lot of variations. The recently synthesized one $\left\{\mathrm{Mn}(\mathrm{hfac})_{2}\right\}_{3}(3 \mathrm{R})_{2}$ (Markosyan et al. 1998) has been investigated to realize the $(5 / 2,3 / 2)$ spin chain. We hope the present calculations will stimulate not only further theoretical investigations, but also experimental explorations into the magnetization plateaux in ferrimagnets as spin-polymerized materials.

\section{ACKNOWLEDGMENT}

The authors thank Dr. K. Okamoto for useful discussion. This work is supported by the Japanese Ministry of Education, Science, and Culture through Grant-in-Aid No. 11740206 and by the Sanyo-Broadcasting Foundation for Science and Culture. The computation was done in part using the facility of the Supercomputer Center, Institute for Solid State Physics, University of Tokyo.

Yamamoto S 2000 Phys. Rev. B 61 842, and references therein

Oshikawa M, Yamanaka M and Affleck I 1997 Phys. Rev. Lett. 781984

Totsuka K 1998 Phys. Rev. B 573454

Tonegawa T, Nakao T and Kaburagi M 1996 J. Phys. Soc. Jpn. 653317

Cabra D C, Honecker A and Pujol P 1997 Phys. Rev. Lett. 795126

Cabra D C and Grynberg M D 1999 Phys. Rev. Lett. 82 1768

Sakai T and Takahashi M 1998 Phys. Rev. B 573201

Narumi Y, Hagiwara M, Sato R, Kindo K, Nakano H and Takahashi M 1998 Physica 246-247 509 
Shiramura W, Takatsu K, Kurniwan B, Tanaka H, Uekusa H, Ohashi Y, Takizawa K, Mitamura and Goto T 1998 J. PHys. Soc. Jpn. 671548

Yamamoto S and Sakai T 1999 J. Phys.: Condens. Matter 115175

Sakai T and Yamamoto S 1999 Phys. Rev. B 604053

Kuramoto T 1998 J. Phys. Soc. Jpn. 671762

Cardy J L 1984 J. Phys. A 17 L385

Blöte H W, Cardy J L and Nichtingale M P 1986 Phys. Rev. Lett. 56742

Affleck I 1986 Phys. Rev. Lett. 56746

Okamoto K and Nomura K 1992 Phys. Lett. A 169433

Nomura K 1995 J. Phys. A 285451
Kahn O 1987 Struct. Bonding (Berlin) 6889

Kahn O, Pei Y and Journaux Y 1995 in Inorganic Materials, edited by Bruce D W and O'Hare D (Wiley, New York) 95 Drillon M, Coronad E, Beltran D and Georges R 1985 J. Appl. Phys. 573353

Drillon M, Coronado E, Beltran D, Curely J, Georges R, Nugteren P R, De Jongh L J and Genicon J L 1986 J. Magn. Magn. Mat. 54-57 1507

Caneschi A, Gatteschi, Renard J -P, D, Ray P and Sessoli R 1989 Inorg. Chem. 28 1976; 2940

Markosyan A S, Hayamizu T, Iwamura H and Inoue K 1998 J. Phys. Soc. Jpn. 102323 